\title{
Civilisations
}

Revue internationale d'anthropologie et de sciences

humaines

49 | 2002

Pain, fours et foyers des temps passés

\section{Pain, pâtisserie et religion en Europe Pré- et Protohistorique}

Origines et attestations cultuelles du pain

\section{Max Währen}

\section{OpenEdition}

\section{Journals}

Édition électronique

URL : http://journals.openedition.org/civilisations/1822

DOI : 10.4000/civilisations. 1822

ISSN : 2032-0442

Éditeur

Institut de sociologie de l'Université Libre de Bruxelles

\section{Édition imprimée}

Date de publication : 3 juin 2002

Pagination : 381-400

ISBN : 0009-8140

ISSN : 0009-8140

Référence électronique

Max Währen, « Pain, pâtisserie et religion en Europe Pré- et Protohistorique », Civilisations [En ligne], 49 | 2002, mis en ligne le 01 juin 2005, consulté le 02 mai 2019. URL : http://journals.openedition.org/ civilisations/1822 ; DOI : 10.4000/civilisations.1822

Ce document a été généré automatiquement le 2 mai 2019.

(c) Tous droits réservés 


\title{
Pain, pâtisserie et religion en Europe Pré-et Protohistorique
}

Origines et attestations cultuelles du pain

\author{
Max Währen
}

\section{NOTE DE L'ÉDITEUR}

L'article qui suit constitue un inédit riche en données d'un grand intérêt pour notre sujet d'étude. Pour cette raison notre comité de lecture, qui a préféré que ce texte ne lui soit pas soumis, en a néanmoins approuvé la publication dans ce volume.

Given the richness of the material presented, the following article will certainly be of great interest to anyone studying the specific topic of the archaeology of bread. It has been published without the editorial board conecting it, but with its approval.

\section{NOTE DE L'AUTEUR}

Vu la concision du présent article, nous n'avons évidemment pu étudier l'ensemble du sujet.

Pour finir, j'aimerais remercier la grande ethnobotaniste Dr. Maria Hopf et l'archéologue tout aussi méritante Dr. Emma Pressmar auxquelles je suis lié par plusieurs décennies de collaboration. Dans le domaine plus large de la recherche sur le pain, je voudrais également faire parvenir mes pensées reconnaissantes à mon ami le Prof. Dr. Ernst Burgstaller. 


\section{Ancêtres et origine du pain en Europe}

1 La signification du pain, de la pâtisserie et des pratiques cultuelles ne peut se comprendre que si l'on sait ce qu'est le pain, quand et comment il est né, et quelle signification il avait à l'époque. Du point de vue géographique, il est inséparable du Proche-Orient, et plus précisément du Croissant Fertile, région située entre la Tigre et l'Euphrate.

2 Le pain trouve son origine lointaine dans la présence au Proche-Orient de grandes plantes herbacées sauvages qui n'existaient pas en Europe. Dans le Croissant Fertile, l'orge, l'amidonnier et l'engrain, tous trois à l'état sauvage, s'étaient acclimatés dans le Nord de la Palestine et en Israël. Il y a plus de 15000 ans, dans un site près d'Ain Gev, on a retrouvé des lames de faucilles, des meules et des mortiers. Cela signifie que les habitants mangeaient des céréales sauvages dont on sait qu'elles sont amères et indigestes quand elles sont crues. Selon nous, les céréales sauvages étaient broyées à la meule et donnaient un peu de farine. Celle-ci était mixée, aplatie en galette et grillée ou cuite.

3 L'expérimentation a permis de déterminer la chaleur nécessaire à cette opération. Peter M. Rudin, sous-directeur de l'école de boulangerie et de pâtisserie Richemont à Lucerne, a découvert avec son chef de laboratoire, D. Jakob, que la cuisson de ces premiers pains demandait environ $260^{\circ} \mathrm{C}$. Cette chaleur permettait d'obtenir un aliment qui se conservait bien et permettait aussi d'adoucir son goût. La forte mastication rendait la production de salive suffisante pour transformer la masse mastiquée en dextrose dans l'appareil digestif et l'injecter immédiatement dans le circuit sanguin comme source d'énergie (aujourd'hui, 15000 ans plus tard, les médias font de la publicité pour le dextrose). L'homme découvrit rapidement la valeur de ces céréales sauvages, les soigna et les transplanta dans une terre plus fertile. La culture permit ainsi d'obtenir des céréales plus grandes et de meilleure qualité.

Fig. 1 : Surface supérieure d'un pain de froment dont la datation est assurée par la dendrochronologie, 3719 à 3699 av. J.-C. (Suisse, Montmirail, Ct. de Neuchâtel). Il présente de nombreuses particularités qui en font une pièce unique. Identification : Max Währen. Photo : Jürg Währen.

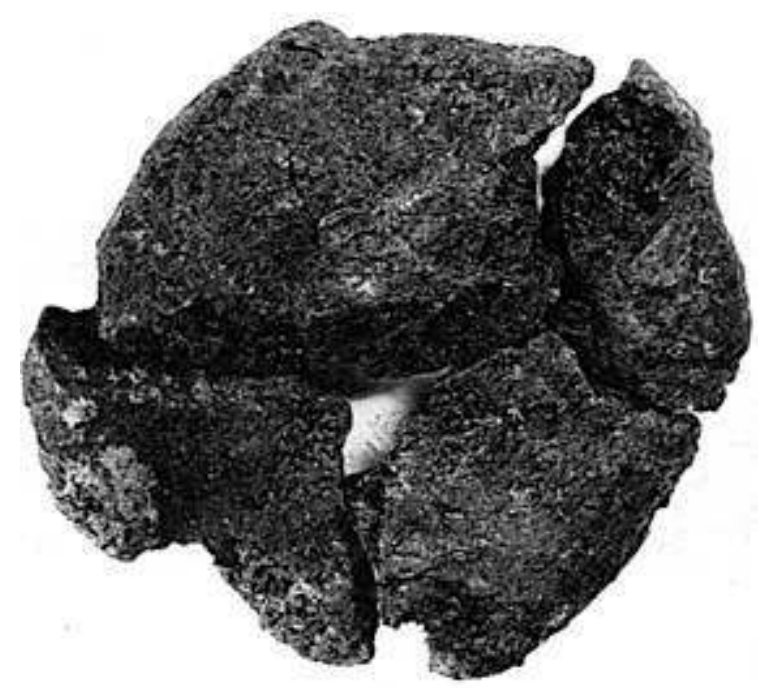

Quand le Néolithique atteignit l'Europe au départ du Proche-Orient, il introduisit la culture des céréales et la galette grillée et cuite. Mais le pain véritable n'apparaît que 
pendant le $\mathrm{V}^{\mathrm{e}}$ millénaire en Europe, à différents endroits marqués par la culture néolithique. On peut ainsi mentionner que la cuisson du pain était déjà connue en Yougoslavie vers 4850/4700 avant J.- C. (site de Lepenski Vir IIIa/b, culture de Starcevo) et en Suisse vers 4300 avant J.-C. (site d'Egolzwil III). La plus ancienne attestation de pain in natura étudiée par l'auteur (site de Montmirail) date de 3719 à 3699 avant J.-C. (Fig. 1 et 2).

Fig. 2 : Surface inférieure du pain représenté à la figure 15 qui présente partiellement des fissures intéressantes pour comprendre le mode de cuisson. Photo : Jürg Währen.

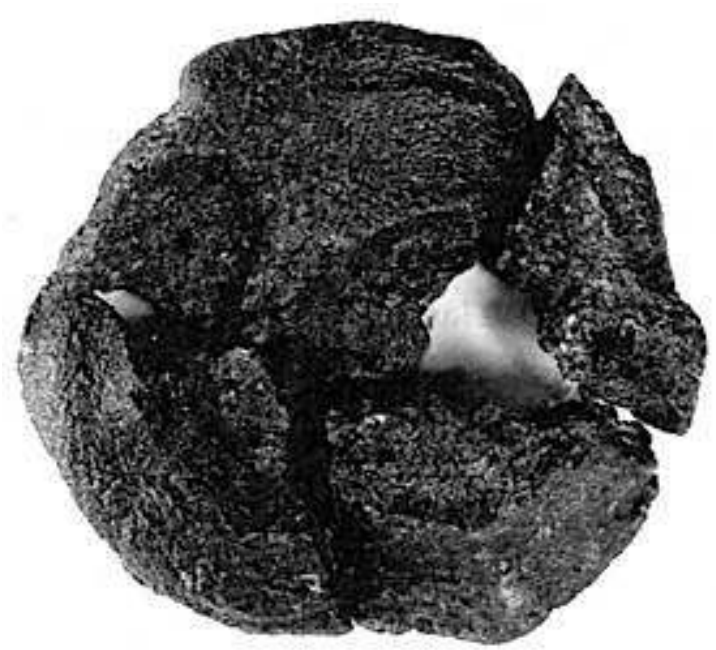

5 Nos identifications des pains de Douanne (Suisse, 3560 à 3530 avant J.-C., Néolithique moyen) s'étendant de la région Rhône-Alpes des contrées au Jura suisse, ainsi que des morceaux de pain d'Ouroux (Saône-et-Loire, Âge du Bronze) témoignèrent la première fois de cette évolution. En ce qui concerne l'Europe centrale et occidentale, ce que l'on entend communément par pain n'a donc pas été inventé à un endroit particulier, mais se retrouve dans plusieurs sites du Néolithique moyen (pour simplifier les choses, dans une bande qui s'étend de l'embouchure du Rhône au Bas-Rhin).

6 Nous avons pu déterminer précisément l'ensemble de l'évolution grâce à l'identification de trouvailles faites dans divers sites et s'étalant sur une période de plus de 3800 ans. Hans Grütter, archéologue du canton de Berne, nous a permis d'étudier les objets découverts dans divers sites du début du $4^{\mathrm{e}}$ au $3^{\mathrm{e}}$ millénaire. C'est ainsi que nous avons $\mathrm{pu}$ identifier le plus vieux pain européen parfaitement conservé et l'analyser dans les détails. Il s'agit du pain de Douanne (Lac de Bienne). Ce pain date de 3560 à 3530 avant J.-C., il a été fabriqué à partir de froment finement moulu et de levain, et présente une jolie courbure, comme on peut le voir sur la figure 3. Sa forme et sa fabrication ne permettraient pas de le distinguer du pain levé qui existe encore dans les Alpes, notamment dans le Valais. 
Fig. 3 : Le plus vieux pain du monde entièrement conservé du néolithique occidental, 3560-3530 av. J.-C. Dans les tumulus, il ne s'agit pas de grain mais de pain ratatiné. Froment finement moulu, à l'état frais, il y a 5500 ans : diamètre, environ $17 \mathrm{~cm}$, hauteur : 4,5 à $5 \mathrm{~cm}$, poids, environ $330 \mathrm{~g}$. Trouvé à Douanne (canton de Berne) culture Cortaillod franco-suisse. Identification et publication Max Währen. Photo : Jürg Währen. Collection du service archéologique du canton de Berne

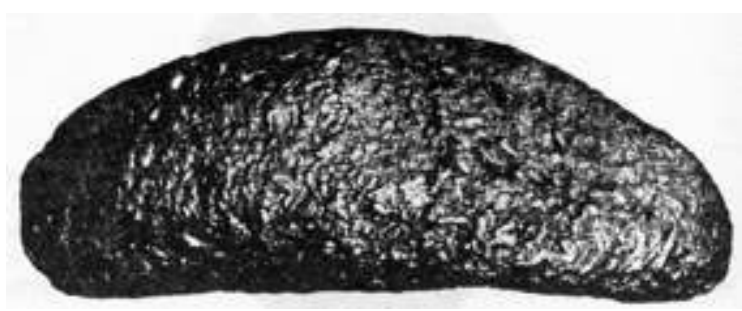

7 Il nous paraît utile de replacer dans l'évolution les résultats de ces recherches, que l'auteur a prouvé par des analyses détaillées.

Issue du Midi, la Culture Chassey et ses sphères d'influence se sont notamment étendues jusqu'aux actuels lacs du Jura. Elle a pris le nom de Culture Cortaillod, d'après le site du même nom, sur le lac de Neufchatel et aurait apporté à Douanne, sur le lac de Bienne, dans les années 3550 avant J.-C. l'art complet de la cuisson du pain, mais à côté de l'attestation du pain aucun indice d'existence de « fine » boulangerie ou pâtisserie n'a été trouvé.

9 Vers 3300 avant J.-C., la Culture de Horgen (d'après du site de Horgen sur le lac de Zurich) s'étendit de l'Est jusqu'aux lacs du Jura et se mêla à Montilier (lac de Morat) à la Culture Cortaillod. Nous avons étudié tout ce qui y a été découvert en matière de céréales et avons pu constater la présence d'une variété insoupçonnée de pâtisseries raffinées, datées entre 3178 et 3118 avant J.-C. Attribuées à env. 3150 avant J.-C., nous avons identifié et étudié trois sortes de pain, mais aussi au moins neuf sortes de pâtisseries, dont quelques exemples seulement sont mentionnés ci-après :

- 1) Les barquettes

- 2) Les tartelettes rondes, faites de farine de froment finement moulue, ayant un fond de pâte très mince, un rebord de $15 \mathrm{~mm}$ de haut et une épaisseur de $2 \mathrm{~mm}$ (ce qui correspond à nos tartelettes aux fraises actuelles).

- 3) Les tartes rappelant nos tartelettes alsaciennes.

- 4) Coupelles de pâte rondes, façonnées sur des bandes d'écorces de bouleau de $4 \mathrm{~cm}$ de long ; telle la plaque de cuisson d'un four actuel, celles-ci laissaient irradier doucement et progressivement la chaleur de la cuisson, comme il se doit pour des pâtisseries.

Fig. 4 : Cette pâtisserie cuite sur écorce de chêne est également une pièce unique pour la préhistoire. Elle est datée par dendrochronologie de 3178 à 3118 av. J.-C. (Suisse, Montilier, Lac de Morat, Ct. de Fribourg). Identification : Max Währen. Photo : Jürg Währen.

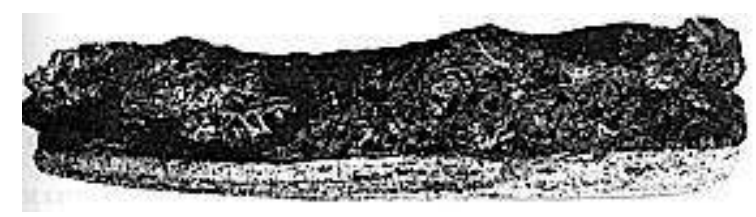

- 5) L'écorce de chêne a servi à une pâtisserie plus légère, car plus riche en blanc d'œuf ; on savait donc exactement quelle sorte d'écorce convenait comme plaque à quelle pâtisserie. 
Fig. 5 : Cette pâtisserie au miel a été cuite sur écorce de bouleau (ici la face de cuisson). Le miel remplissait une cuvette sur la surface supérieure. Elle est datée par dendrochronologie de 3178 à 3118 av. J.-C. (Suisse, Montilier, Lac de Morat, Ct. De Fribourg). 64 x 67,4 mm. Identification : Max Währen. Photo : Jürg Währen.

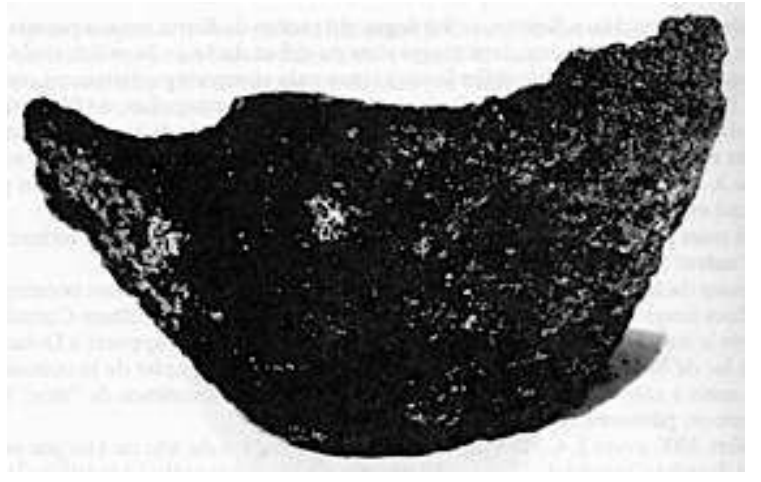

Fig. 6 : Pâtisserie en forme de barquette. Amidonnier. Elle est datée par dendrochronologie de 3178 à 3118 av. J.-C. (Suisse, Montilier, Lac de Morat, Ct. De Fribourg). 61,1 x 45,34 x 36 mm. Identification : Max Währen. Photo : Jürg Währen.

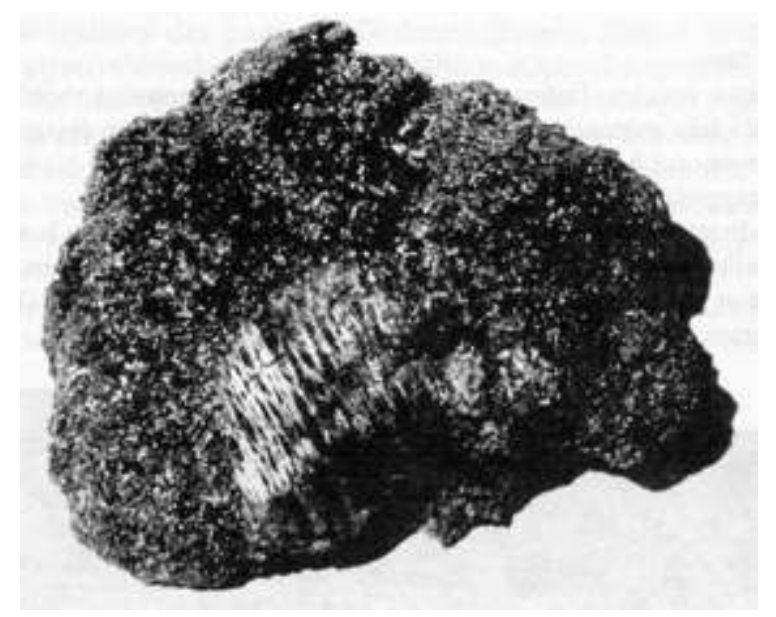

- 6) La plus étonnante de ces pâtisseries vieilles de 5150 ans, nous l'avons appelée la « brioche de l'Age de pierre ». L'auteur a reçu cet objet dans son état d'origine ; c'était alors une motte sale, carbonisée, d'origine inconnue et recouverte de calcaire. Après nettoyage, elle se révéla être le gâteau le plus raffiné connu pour la préhistoire, d'une longueur de 92,3 mm, d'une largeur de 73,3 mm, d'une hauteur de 52,2 mm et d'un poids de 45,75 g. Elle avait un côté bombé et l'autre plat. Nous avons supposé que la pâte avait dû être pressée dans un moule à gâteaux. Des clichés radio confirmèrent irréfutablement qu'un moule carré aux arêtes finement travaillées avait été utilisé et que le côté bombé ne s'était formé que par la suite. Voilà comment fut découvert le plus vieux moule à gâteau du monde. 
Fig. 7 : Pâtisserie avec pierre chauffante ou moulante, la cuvette permettant d'y placer des baies. La pâte a été montée à la main en formant des marches et en laissant des empreintes de paume et de doigts. Elle est datée par dendrochronologie de 3178 à 3118 av. J.-C. (Suisse, Montilier, Lac de Morat, Ct. de Fribourg). 72,24 x 74,34 mm. Identification : Max Währen. Photo : Jürg Währen.

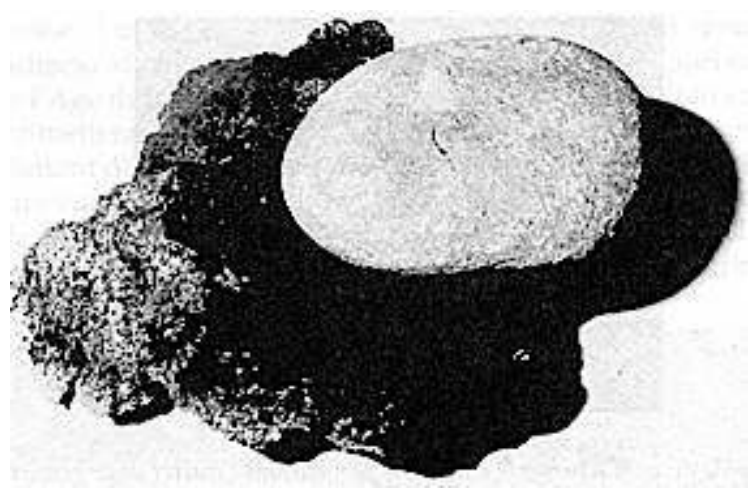

Fig. 8 : Cette pâtisserie avec pierre chauffante enfoncée dans sa masse est probablement la pièce la plus intéressante et rare connue pour la préhistoire du pain. La pierre ne servait pas seulement à donner de la chaleur, mais aussi comme thermomètre et pour façonner une cuvette.Elle est datée par dendrochronologie de 3178 à 3118 av. J.-C. (Suisse, Montilier, Lac de Morat, Ct. de Fribourg, Inv. $\mathrm{N}^{\circ}$ MU-PLA 79, SE D 475/102, FS 2)

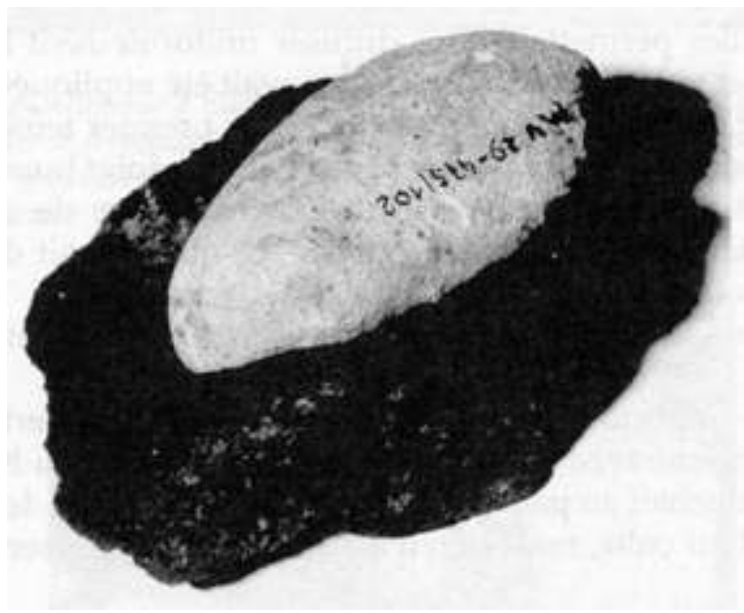

Sur le côté cuit, nous avons détecté des encoches de la forme d'une croix de Saint André qui se prolongent jusqu'à un centimètre sous la surface. Si une encoche avait été plus longue de 2 à $3 \mathrm{~mm}$, le gâteau se serait fendu. Nous avons ensuite noté que les entailles permettaient de diffuser uniformément la chaleur dans ce gâteau assez haut et volumineux. A la surface avait été appliquée une pierre chauffante qui avait fait office de thermomètre dans un premier temps : on mesurait le degré de chaleur nécessaire en y posant brièvement le doigt humecté par la langue. Nous avons ainsi découvert le plus ancien thermomètre de cuisson. On aurait ensuite retiré la pierre préalablement graissée, ce qui formait dans le gâteau une sorte de coupelle, que l'on pouvait remplir de friandises. 
Fig. 9 : Radiographie du pain représenté à la figure 6, montrant l'intérêt de ce genre d'analyse. La partie inférieure claire, à rebords aigus, montre que la pâtisserie, aujourd'hui bombée, a été enfoncée dans un moule lors de sa fabrication. Les rebords supérieurs assez aigus prouve la même chose. La croix d'Andréas incisée apparaît comme une ombre. En haut et en bas à droite on aperçoit de rares petits débris de pierres qui proviennent de la meule. Les surfaces sont régulières et témoignent d'une farine finement moulue, d'une bonne levée de la pâte et d'une cuisson en profondeur. Une technologie boulangère d'un haut niveau est ainsi attestée. Etude : Max Währen. Rayons X: 100 milliampères, 20 milliampères seconde, 48 kilovolts.

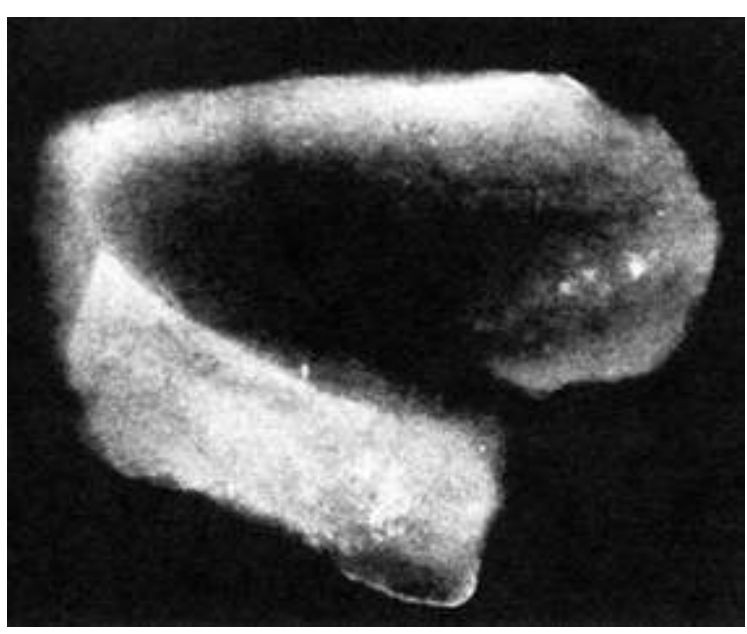

D’autres formes de pâtisserie du Néolithique comprenaient notamment le gâteau au miel.

Il m'a semblé important de rapporter toutes ces découvertes, parce que l'on ignorait jusqu'à présent avec quel raffinement les hommes du Néolithique maîtrisaient tout ce qui touchait au pain et à la pâtisserie. Une partie de cette maîtrise doit se retrouver dans leur culte, mais rien n'a été prouvé directement à ce sujet.

\section{Le pain et la religion au Néolithique et à l'Age du Bronze}

Dans le culte néolithique, le moulin, qui servait à faire de la farine fine pour la confection du pain et de la pâtisserie, acquit une signification sans précédent en se substituant au pain comme offrande aux dieux et comme cadeau funéraire (Fig. 10). L'homme de l'Age de Pierre savait naturellement que la pierre était un objet durable et immuable. Est-ce pour cette raison qu'il plaçait des meules dans les tombes pour permettre de faire du pain dans l'au-delà? Il s'agissait de petites meules à main, d'environ $6 \mathrm{~kg}$, faciles à porter et moulant finement la farine pour faire d'excellents pains ou pâtisseries (intervenant eux aussi dans les rites funéraires?). Sur base d'autres parallèles, nous considérons que la meule symbolisait peut-être un pain ou une pâtisserie de qualité. 
Fig.10 : Meule datant du Néolithique d'Europe occidentale 3560-3530 av. J.-C. Longueur : 40,5 cm. A gauche, manche se terminant en pointe. Cette meule était actionnée en position assise, jambe écartées, et produisant de la farine fine. Elle servait de préférence d'offrande cultuelle funéraire. Photo : Jürg Währen, nouvelle collection Max Währen.

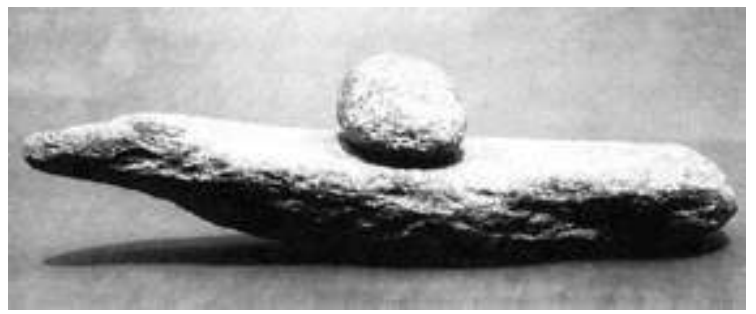

Le don d'une meule impliquait une cérémonie religieuse. Lors de fouilles dans un site néolithique d'Allemagne (Auschwitz /Weideroda, Kreis de Borna) on a retrouvé le crâne d'un jeune homme reposant sur une demi-meule. En Suisse romande (Baulmes / Mistredame, canton de Vaud), les archéologues ont mis au jour une tombe de l'Age du Bronze dans laquelle la tête du défunt était entourée d'une meule brisée rituellement en deux (Fig. 11). De cette manière, la meule - substitut du pain permettant de le renouveler indéfiniment - entourait la bouche et la tête. Il existe une autre variante à la fin de l'Age du Bronze: l'urne cinéraire est renversée sur une meule (Allemagne, Hesse, Kreis de Friedberg, Gambach). Ici aussi, un rite souligne le lien entre le moulin qui donne du pain et les cendres du mort.

Fig. 11 : Dépôt de crâne humain entre deux parties d'une meule brisée rituellement. Age du Bronze Suisse, Mistredame, districte d'Orbe.(d'après Jahrbuch der Schweizer Gesellschaft für Urgeschichte 53, 1966/67).

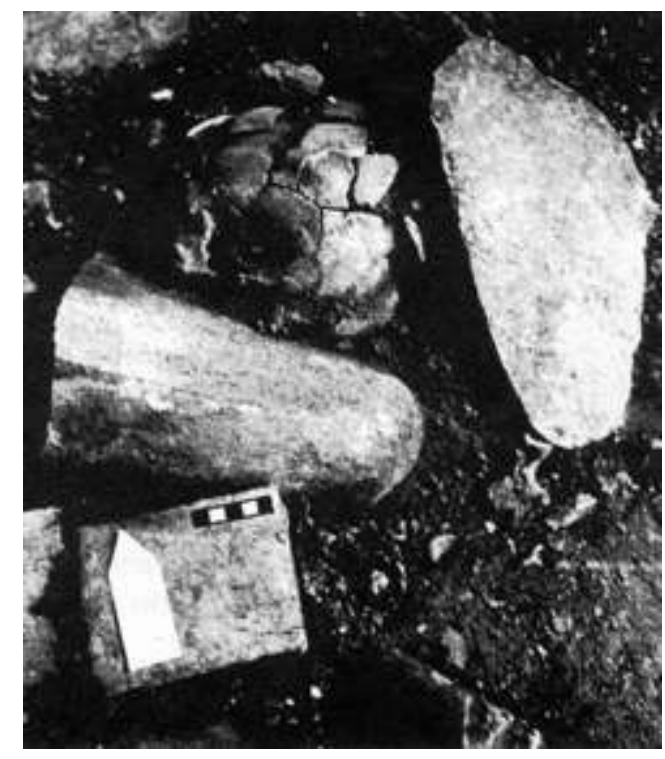

\section{Le pain et la religion à la fin de l'Age du Bronze et au premier Age du Fer}

Nous arrivons ainsi à la civilisation des Champs d'Urnes - caractérisée par des nécropoles d'urnes cinéraires - qui va de $1400 / 1300$ à 700 avant J.-C. Elle coïncide avec la fin de l'Age du Bronze et les deux derniers siècles appartiennent déjà à l'Age du Fer dans certaines 
régions. Le pain a une signification plus grande et la construction de fours collectifs dans le village crée une communauté autour de la fabrication du pain. Cela se traduit également dans la religion.

La culture indo-européenne des Hittites, qui fonda un empire en Asie Mineure à l'époque correspondant à l'Age du Bronze en Europe, présente plusieurs caractéristiques qui pourraient rappeler le culte de l'époque des Champs d'Urnes. Ainsi, le four y avait également une signification cultuelle ; il était quasi considéré comme sacré puisque c'est devant lui que le soldat hittite prêtait serment.

17 L'archéologue Emma Pressmar, d'Ulm, a fait une découverte unique dans le site d'Elchinger Kreuz (Allemagne, Kreis de Neu-Ulm, Fig. 12 et 13).

Fig. 12 : Vue en plan d'un four à pain et d'un dépôt cultuel, situé à la limite de celui-ci et de la fosse de rejet des cendres. Le dépôt est composé d'ossements de boeufs qui ont été ensevelis à proximité de l'ouverture avant du four (Allemagne, Elchinger Kreuz, Kreis Neu-Ulm, reproduit avec l'accord de l'archéologue, Dr. Emma Pressmar). Une fonction de protection et apotrophaïque pourrait ici être invoquée d'après l'archéologue.

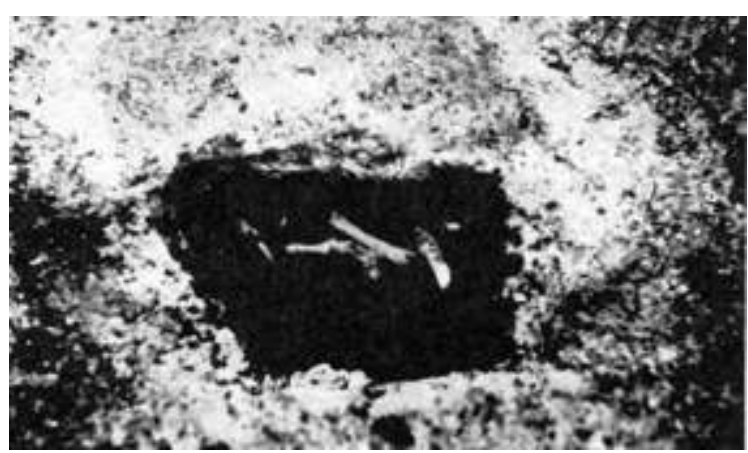

Fig. 13 : Vue de la base de la fosse de rejet des cendres de la figure 10 et de son dépôt cultuel. (Allemagne, Elchinger Kreuz, Kreis Neu-Ulm, reproduit avec l'accord de Dr. Emma Pressmar).

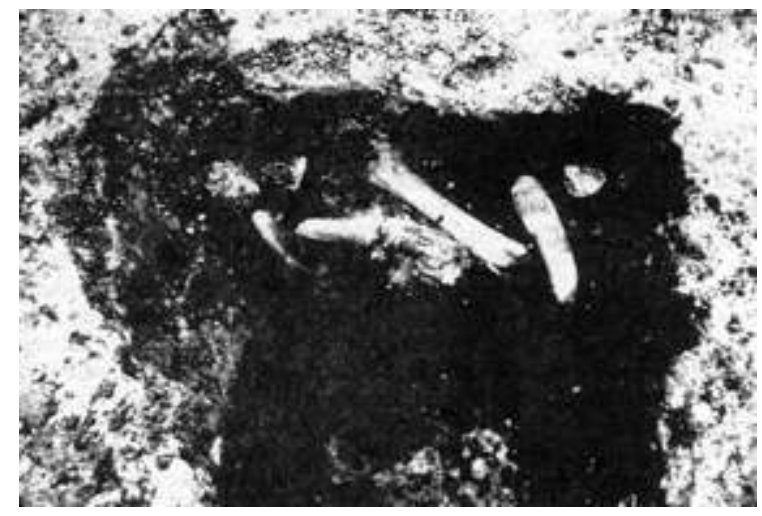

18 Elle a trouvé, sous un four à pain, les restes d'un jeune bovin abattu qui correspondent à un animal sacrifié. Elle en conclut que ces dépôts cultuels du four avaient une fonction à la fois de garde et d'avertissement. Il nous semble que ce four à pain avait un caractère religieux, en plus de son aspect matériel, et servait de lieu de sacrifice.

19 L'urne funéraire de Montescudaio (Musée archéologique de Florence, VII ${ }^{\mathrm{e}}$ siècle avant J.C., Fig. 14) présente une autre signification cultuelle du pain. Le sommet du couvercle représente le défunt devant une table dressée où se trouve du pain, et, à côté, une figure humaine dont la partie inférieure est transformée en urne. Il y avait donc une identification du mort avec le pain prometteur de vie éternelle ou, en d'autres termes, le 
mort et le dieu ne font plus qu'un grâce au pain. Pour le mort, il ne s'agirait pas simplement d'une représentation mais d'une réalité de l'au-delà conditionnée par le culte.

Fig. 14 : Urne à incinération montrant un boulanger et son four (au milieu à gauche), le défunt près d'une table avec des pains (en haut sur le couvercle). A côté de lui on aperçoit un personnage avec la cuve permettant de pétrir la pâte. Il s'agit d'une des attestations les plus intéressantes de l'offrande funéraire du pain. Civilisation étrusque, $7^{\mathrm{e}}$ siècle av. J.-C. (Italie, Montescudaio).

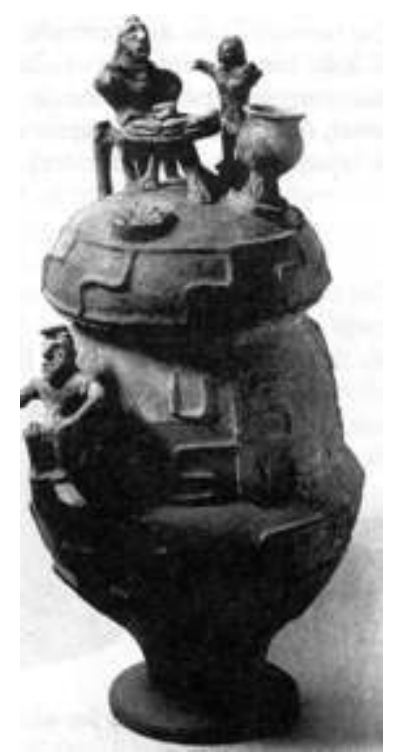

La relation entre le pain et la vie dans l'au-delà est illustrée par les restes d'os contenus dans une urne de l'époque des Champs d'Urnes qu'Emma Pressmar a retrouvé (à Bellenberg). Nous avons pu retrouver des parties de pain levé dans les minuscules cristaux osseux. Le pain a donc été déposé sur le cadavre avant la crémation. Il s'agissait d'une offrande à la divinité et d'une nourriture pour l'éternité. On retrouve ces caractéristiques du culte des morts chez les Hittites, dont nous avons également examiné des restes de pain. Dans le culte hittite, la fraction et l'émiettement rituels du pain (offrande et aliment pour l'au-delà) avaient la plus haute signification, de même que l'opposition entre pain doux ou sûr. Le pain doux consiste en un gâteau et le pain sûr en un pain levé.

21 Cette tradition se retrouve dans de nombreux sites de l'époque des Champs d'Urnes, à partir d'environ 1300 avant J.-C., en Europe occidentale. Dans cette région géographique, pour toute la durée de l'époque, nous avons identifié 300 morceaux de pain et de gâteaux, qui ont été placés dans les urnes ou dans les tombes. Dans $89 \%$ des cas, il s'agissait de très bon pain ou de gâteaux. Actuellement, les morceaux ont un diamètre de $1 \mathrm{~cm}$. Lorsqu'ils étaient frais, il devait être d'environ $2,5 \mathrm{~cm}$. Nous avons refait l'expérience et découvert que le pain était placé dans l'urne avec le pouce, l'index et le majeur. Etant données les fabrications différentes, nous avons constaté que plusieurs personnes de la communauté en deuil devaient offrir du pain. Ce culte était également pratiqué à l'Age du Fer préromain (voir plus loin).

Nous avons fait une découverte cultuelle très intéressante dans un site datant de 900 à 600 avant J.-C. (Allemagne, Overgönne, Kreis de Stade, Fig. 15 et 16). Dans un dépôt cultuel, nous avons identifié la moitié d'un pain, pointu aux deux bouts, marqué par une entaille en forme de vague et par deux cavités. Il s'agit de la forme la plus ancienne de 
pain cultuel découverte en Europe. Le pain entièrement carbonisé mesure $7 \mathrm{~cm}$; à l'état frais, c'est-à-dire voici environ 2700 ans, il devait mesurer 17 à $18 \mathrm{~cm}$ de long. Des radiographies et d'autres examens nous ont permis de découvrir une petite pièce de métal à l'intérieur. Ce pain, armé d'un morceau de métal, était vraisemblablement une offrande propitiatoire à une force de la nature divinisée (peut-être la foudre).

Fig. 15 : Le plus vieux pain ayant une forme, 900-600 av. J.-C. (Allemagne, Ovelgönne, Kreis de Stade), dans lequel se trouve une petite offrande de métal. Il provient d'une fosse cultuelle. II s'agit de la moitié d'un pain dentelé en forme de coin, qui rappelle l'attribut d'un dieu de la foudre indoeuropéen. Identification et dessin de restruction : Max Währen. Photo : Jürg Währen. Musée archéologique de Hambourg.

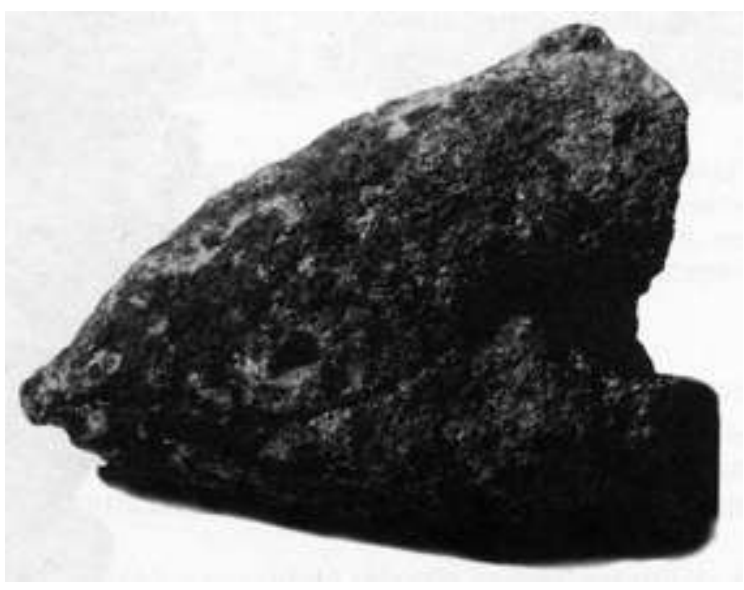

Fig. 16 : Reconstruction d'un pain entier que l'on fabrique encore dans la plupart des pays européens

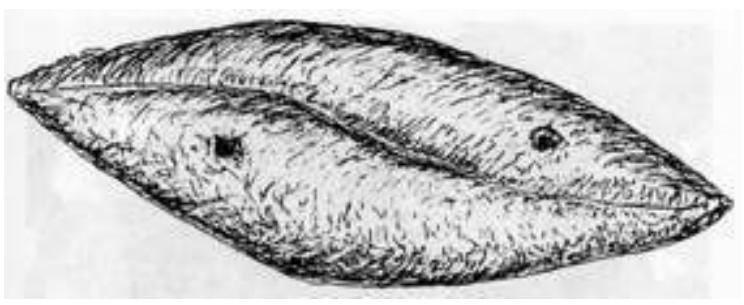

Metzler a trouvé sous le chemin de bois qui traverse le Marais d'Ipweg (Allemagne, près de Moorriem, Kreis de Wesermarsch) une galette de pain en bon état datant du début de l'époque de Hallstatt, vers de 713 avant J.-C. Selon la première hypothèse, il s'agissait d'un pain oval coupé avec une sorte de couteau-scie. En examinant cet objet, nous sommes arrivés à la conclusion que la pâte nécessaire à la cuisson d'une galette ronde d'un diamètre de $15 \mathrm{~cm}$ a été coupée un peu au-dessus de la moitié, que les coupures ont été pressées au moyen d'un morceau de bois plat et ce n'est qu'alors qu'un demi pain au moins a été cuit.

Le pain du Marais d'Ipweg se compose en majeure partie de cire d'abeille, révèle des traces d'épeautre et d'orge ainsi que des graines de millet. Il semble s'agir d'un pain de substitution, destiné à remplacer le vrai pain. On a supposé qu'un pain complet aurait été détruit dans le marais. Nous en déduisons qu'il joua à l'époque un rôle d'offrande pour que soit mené à bien et protégé éternellement l'aménagement du chemin de madriers du marécage. Cela doit être, en Europe, la plus ancienne offrande sous forme de pain, dont le but ait été profane. 
Si ce sujet n'a pas encore été étudié, nous avons a prouvé l'existence de tels pains dans l'ancienne Egypte (M. Währen, Brot im Leben und Glauben des alten Ägypten, ILL. 9). Ce type de demi-pain se trouvait en effet dans la tombe de Touthankamon. Nous en avons également découvert en pâtisserie fine au début du XIII ${ }^{e}$ siècle après J.-C. en Europe centrale et occidentale. Leur seul point commun est leur technique de fabrication. Comme la partie sectionnée n'a pas été pressée trop fortement, l'objet qui nous occupe a formé une croûte plus fine et est devenu agréablement croustillant. Ce détail et la perfection de la fabrication permettent de conclure qu'il ne s'agit pas d'un pain destiné à l'usage courant, mais d'une offrande préparée spécialement. C'est à la fois un remerciement pour la réussite du chemin et une offrande propitiatoire.

\section{Le pain et la religion aux époques celtique et romaine}

Nous avons examiné environ 200 objets provenant de tombes à incinération des époques celtique et romaine, jusqu'au II $^{\mathrm{e}}$ siècle après J.-C. Il s'agit pour l'essentiel de morceaux de pain et de gâteaux destinés aux urnes ou aux tombes contenant l'urne. Les quatre cas les plus exceptionnels sont mentionnées ci-dessous.

Fig. 17 : Confiserie celtique, en forme d'anneau, offrande cultuelle dans une tombe, vers 125 av. J.C. (Allemagne, Wederath-Belginum, Kreis de Bernkastel-Wittlich, Musée du Land de Rhénanie à Trèves). Identification : Max Währen. Photo : Jürg Währen.

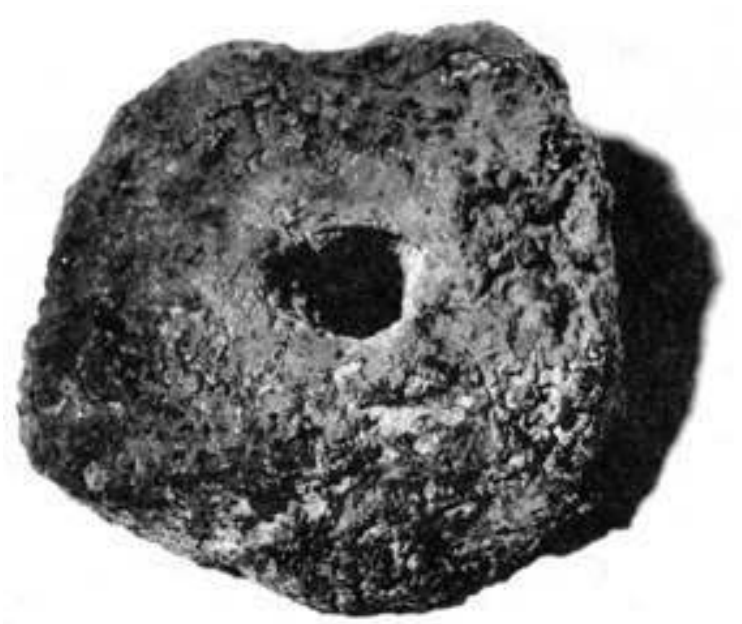

Fig. 18 : Morceau de gâteau en forme de boule, celtique, vers 150-120 av. J.-C. (Allemagne, Wederath-Belginum, Musée du Land de Rhénanie à Trèves). Il s'agit d'une offrande et de la nourriture pour l'au-delà. Identification : Max Währen. Photo : Jürg Währen.

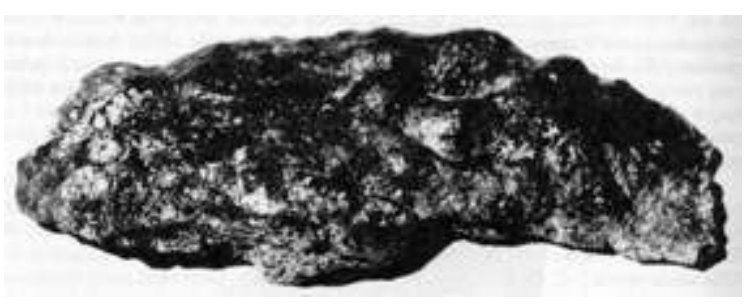


Fig. 19 : Exemple de pain émité ayant une signification cultuelle. Il était utilisé dans la plus grande partie de l'Europe, d'environ 1300 av. J.-C. à l'époque romaine, pour le rituel funéraire. Pour cette photo, des petits morceaux ont été mélangés à des morceaux inhabituellement grands de 2 à $3 \mathrm{~cm}$. Identification et publication : Max Währen. Photo : Jüng Währen. Musée du Land de Rhénanie à Trèves.

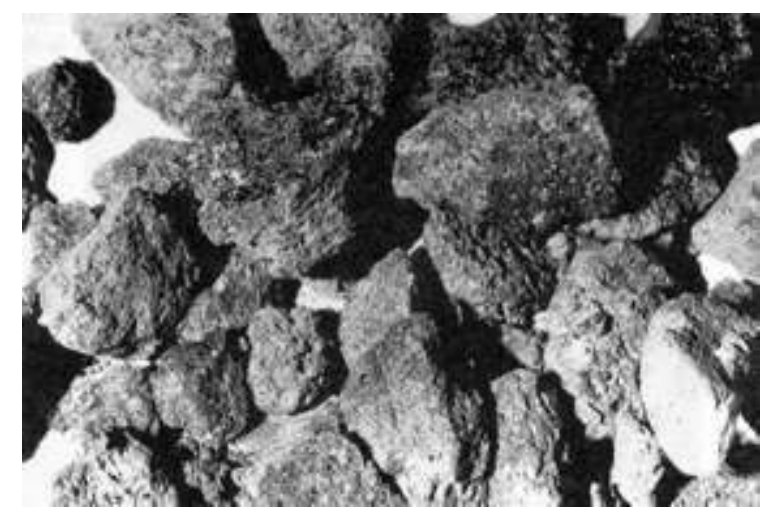

Fig. 20 : Parties extérieures d'un pain votif dont le centre est enfoncé. Epoque romaine, Ile siècle apr. J.-C. (Allemagne, commune de Saffig, Kreis de Mayen-Coblence, Rhénanie-Palatinat). II s'agit d'une offrande de pain typique comme on le connaît à plusieurs endroits (voir aussi fig. 23, ou encore offrandes alimentaires de Stephansfensfel-Brumat, tombe 6 (France, Alsace) et Sarkophage Monticelli (St. Petersburg, Ermitage, Inv. A 433, Währen \& Scheneider 1995)

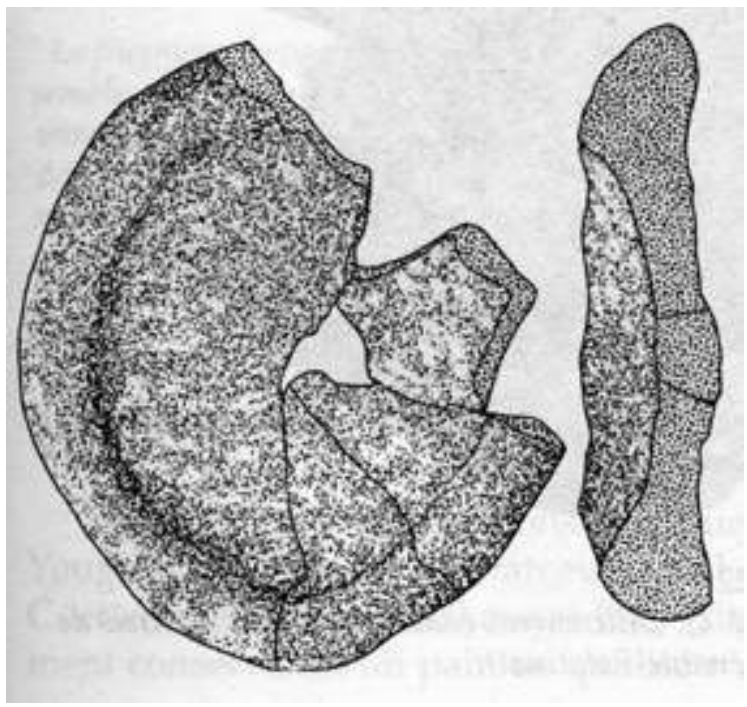

Pour l'époque celtique, il s'agit de deux trouvailles particulièrement importantes dans le territoire trévire à Wederath-Belginum (Allemagne, Kreis de Bernkastel-Wittlich) (Fig. 17-19).

Dans la tombe 84/134, nous avons découvert plusieurs morceaux de gâteau en forme de boule, datant entre 150 et 120 avant J.-C., d'un type inconnu jusqu'ici en Europe préhistorique. Ce gâteau non émietté a été placé dans la tombe qui contenait l'urne en guise d'offrande alimentaire fine et de nourriture pour l'au-delà. Dans le deuxième cas, nous avons identifié dans la tombe crématoire 1700 un petit anneau de confiserie qui est également le seul exemplaire pré- et protohistorique découvert jusqu'à présent en Europe. Il est possible qu'il s'agisse d'autre chose qu'un simple «dessert» donné en offrande. Les pâtisseries cultuelles en forme d'anneau semblent avoir été utilisées depuis toujours. Nous avons attesté qu'il y en avait au $3^{\mathrm{e}}$ millénaire avant J.-C. à Sumer, nous en 
avons trouvé représentées en relief sur un plat funéraire du $2^{\mathrm{e}}$ millénaire avant J.-C. en Crête et il y en avait à Rome, depuis le culte romain jusqu'au début de la chrétienté. Le pain eucharistique a d'ailleurs donné le 'bretzel' au XI ${ }^{\mathrm{e}}$ siècle après J.-C.

Une tombe crématoire du II ${ }^{\mathrm{e}}$ siècle après J.-C. (à Saffig (Allemagne), Kreis de MayenCoblence) est particulièrement intéressante (Fig. 18-20). Nous y avons identifié des fragments de pain d'offrande romain, présentant au centre un creux en forme de coupe dans lequel on a pu déposer des dons alimentaires pour la divinité. Une tartelette aux fruits dont la pâte est creusée de cavités a, quant à elle, pu servir de friandise au défunt dans l'au-delà. On trouve une offrande similaire dans la nécropole de StephansfeldBrumath, Alsace.

Fig. 21 : Dans la même tombe d'incinération que le pain en figure 18 se trouvait ce fond de tarte qui devait jadis être couvert d'une friandise pour l'au-delà. Le fond de tarte été marqué d'encoches pour éviter qu'il n'éclate. Diamètre de 9 à $10 \mathrm{~cm}$, hauteur de 2 à $3 \mathrm{~cm}$, épaisseur de 3,3 à 10,9 $\mathrm{mm}$. Identification : Max Währen. Photo : Jürg Währen.

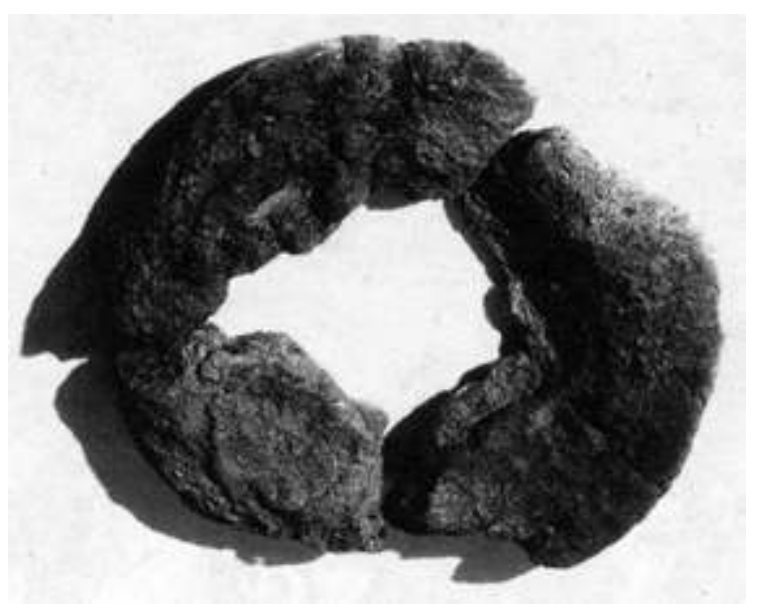

Fig. 22 : Pain d'offrande dans une tombe du II siècle apr. J.-C. (Allemagne, commune de Saffig, Kreis de Mayen-Coblence, Rhénanie-Palatinat).

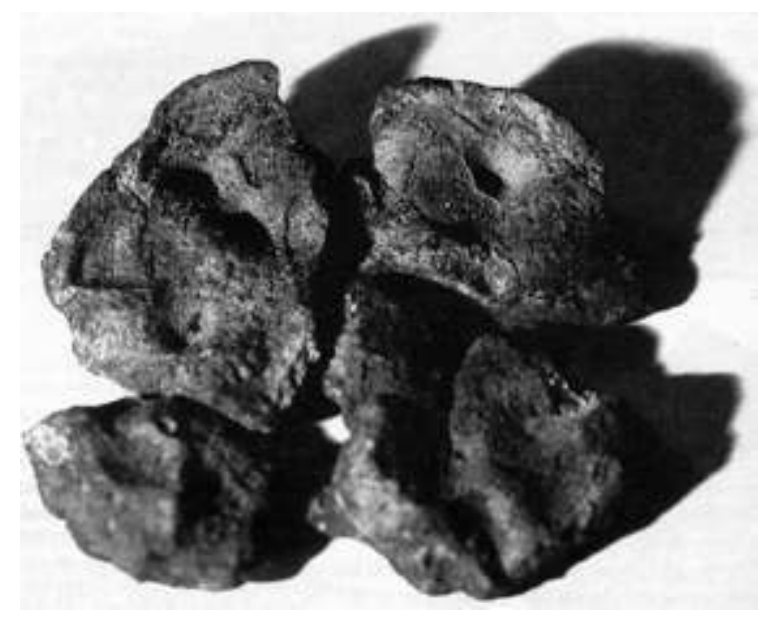


Fig. 23 : Le fragment de pâtisserie issu de la tombe à incinération $n^{\circ} 3$ de Büschdorf (Allemagne, Saarland) semble encore correspondre aux pains d'offrande plus anciens caractérisés par une forme en bol (voir aussi fig. 20). Il est marqué sur sa face supérieure (en bas) par des rebords en relief façonnés à l'aide des doigts. Sur la face de cuisson (en haut), des traces de doigts coïncides avec la mise en place dans un moule semi-circulaire, en forme de feuille. Au centre on peut observer sa structure, sur sa face de cuisson. Diamètre à l'état frais : environ $7 \mathrm{~cm}$. Epoque romaine. Identification : Max Währen. Photo : Jürg Währen.

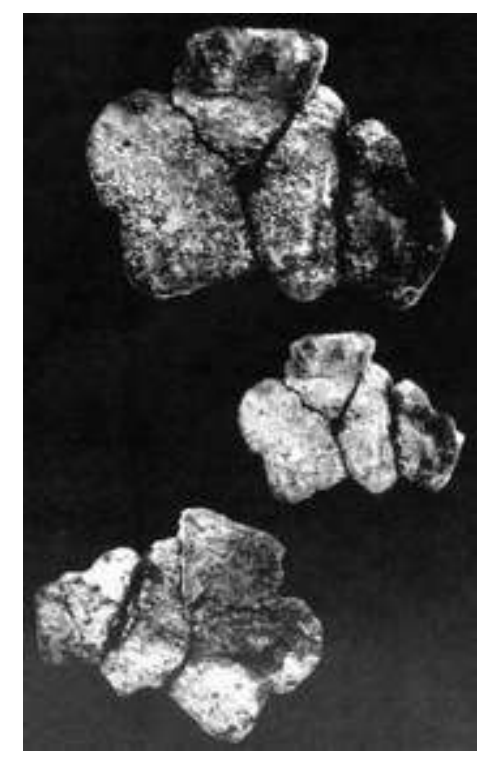

\section{Conclusions}

En résumé, on peut constater que la cuisson du pain était déjà connue en Yougoslavie (Culture de Starcevo, site Lepenski Vir IIIa/b) vers 4850/4700 avant J.-C. et en Suisse vers 4300 avant J.-C. (site de Egolzwil III). Le premier pain entièrement conservé est un pain de qualité supérieure, datant de 3560 à 3530 avant J.-C.. Les premiers essais de pâtisserie proviennent peut-être des colonies établies au bord des lacs du Jura entre 3600 et 3500 avant J.-C., mais cet art connut son plein épanouissement au bord du lac de Morat vers 3150 avant J.-C. 
Fig. 24 : Les célèbres épis d'or de Syracuse sont un dépôt cultuel, peut-être une offrande funéraire, comme cela est attesté pour des branches d'olivier en or. Ils représentent probablement du Triticum compactum. 4 è $3^{\grave{e}}$ s. av. J.-C. (Italie, Syracuse, Sicile, d'après P. Wolters, Festschrift für James Loeb (München 1930)).

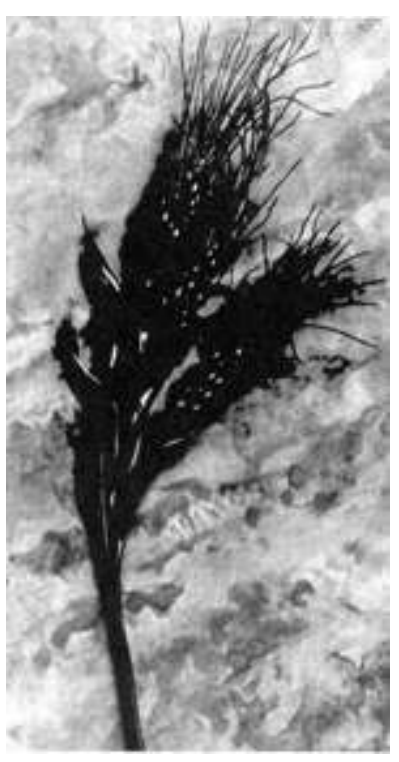

Dans les pratiques cultuelles, le pain et les gâteaux jouent un rôle non négligeable depuis la fin du Néolithique et à l'Age du Bronze. Quelques centaines d'échantillons d'offrande de pains et de restes de gâteaux déposés dans des urnes ou des tombes crématoires sont à l'étude. Ces échantillons de l'époque des Champs d'urnes de Hongrie et d'Europe de l'Est vont encore enrichir notre champ d'investigation.

Etant donné que toutes nos références bibliographiques sont regroupées dans Waehren 2000 et les volumes 25 à 30 de Helvetica Archaeologica (1985-1997) et que toutes les données mentionnées ont été traitées par l'auteur, nous avons renoncé à l'utilisation de renvois à ces références bibliographiques.

\section{BIBLIOGRAPHIE}

Vous trouverez par ailleurs les références de quelques-unes de nos publications dans la bibliographie qui suit.

WAEHREN, M., 1981. Analyse der Brotreste von Telgte a. d. Ems In : Archäologie und Naturwissenschaften II.

WAEHREN, M., 1984. Brote und Getreidebrei von Twann aus dem 4. Jahrtausend vor Christus.

Archäologie der Schweiz / Archéologie suisse 7, n 1 .

WAEHREN, M., 1985. Die Entwicklungsstationen vom Korn zum Brot im 5. und 4. Jahrtausend. Neueste

Untersuchungsergebnisse von Ausgrabungsfunden. Getreide, Mehl und Brot 39 (Bochum 1985), 373-379. 
WAEHREN, M., 1987. Das Brot in der Bronzezeit und älteren vorrömischen Eisenzeit nördlich der Alpen unter besonderer Berücksichtigung von Brotfunden aus Kreisgrabenfriedhöfen des Münsterlandes. Ausgrabungen und Funde in Westfalen-Lippe 5, 23-71.

WAEHREN, M., 1988. Jungsteinzeitliche Speisereste aus dem Kanton Freiburg (Ausgrabungen Portalban, Muntelier, Gletterens). Archäologische Fundberichte 1985. In : Freiburger Archäologie . WAEHREN, M., 1989. Identifizierung von gesäuertem Brot in Knochenasche-Kristallen einer urnenfelderzeitlichen Bestattung in Bellenberg (Ldkr. Ulm) In : Kataloge der prähistorischen Staatssammlung München, Nr. 23 (Kallmünz).

WAEHREN, M., 1989. Brot und Gebäck von der Jungsteinzeit bis zur Römerzeit, Helvetia Archaeologica 20 (Zürich).

WAEHREN, M., 1990. Teig und Feingebäck in der Jungsteinzeit. Neuidentifizierungen, Helvetia archaeologica 84 .

WAEHREN, M., 1990. Brot und Gebäck in keltischen Brandgräbern und römischen Aschurnen Identifizierungen von Brot- und Gebäckfunden aus dem Gräberfeld von Wederath-Belginum, Trierer Zeitschrift 53.

WAEHREN, M., 1990. Die Enträtselung des Brotgeheimnisses der Cheopspyramide. Die zwei bedeutendsten Brotmonumente Ägyptens, Getreide, Mehl und Brot 12, 378-383.

WAEHREN, M., 1992. Krustenuntersuchungen an Kochkeramik. In : FURGER, A.R., DESCHLER-ERB, S, Das Fundmaterial aus der Schichtenfolge beim Augster Theater. Typologische und osteologische Untersuchungen zur Grabung Theater-Nordwestecke 1986/87, Forschungen in Augst 15 (Augst 1992), 447-451.

WAEHREN, M., 1994. Die Urgeschichte des Brotes und Gebäcks in der Schweiz, Helvetia archaeologica 99.

WAEHREN, M., 1994, Fladen, Fladenbrot, In : Reallexikon der Germanischen Altertumskunde (J. Hoops), 9.

WAEHREN, M., 1996. Vorgeschichtliche Brotreste aus der nördlichen Lüneburger Heide, Harburger Jahrbuch 19.

WAEHREN, M., 1997. Das jungsteinzeitliche Brot von Montmirail, Helvetia Archaeologica 28 (Zürich). WAEHREN, M., 2000. Gesammelte Aufsätze zur Brot - und Gebäckkunde und -geschichte. 1940-1999 (Vater und Sohn Eiselen Stiftung/ Deutsches Brotmuseum, Ulm).

WAEHREN, M., DAVIES, R.W, 1978. S.vº. Brot, In : Reallexikon der Germanischen Alterskunde, (Berlin, New York).

WAEHREN, M., SCHNEIDER, C., Die puls. Römischer Getreidebrei. Augster Museumshefte 14 (1995).

\section{RÉSUMÉS}

Faisant le bilan d'une longue carrière consacrée à la recherche sur le pain archéologique, l'auteur nous livre pour la première fois une synthèse de ce qui pourrait être considéré comme des attestations rituelles liées au pain. Après un récapitulatif de l'origine du pain en Europe, il présente les restes d'aliments céréaliers rituels probables qu'il a personnellement pu étudier. De forme et de composition exceptionnelles, ils sont pour la plupart issus d'Allemagne et de Suisse. Il s'agit dans la majorité des cas de la seule attestation de ces rituels que l'on possède aujourd'hui 
au niveau européen. L'interprétation de leur mode de fabrication et de leur utilisation rituelle est confrontée aux résultats de nombreuses expérimentations et de reconstitutions. Des rapprochements avec les connaissances concernant le Proche-Orient et les époques historiques sont proposés.

A long career of research on archaeological bread allows the author to propose the first synthesis on traces of rituals related to bread. After a long introduction on the origin of bread in Europe, he presents supposedly ritual cereal food remains that he has personally studied. Most of these have exceptional shapes or compositions and are from Germany or Switzerland. Most of these remains are unique examples for this ritual in the whole of Europe until now. The interpretation of their method of preparation and of the involved ritual is confronted with the results of several experiments and reconstructions, some confrontation with the authors knowledge about the Near East and historical periods are proposed.

INDEX

Mots-clés : Pain, céréales, pâtisserie, rituel, religion

Keywords : Bread, cereals, pastry, ritual

\section{AUTEUR}

MAX WÄHREN

Bern, Schweiz 